\title{
Immunosuppression by piperine as a regulator of the NLRP3 inflammasome through MAPK/NF-KB in monosodium urate-induced rat gouty arthritis
}

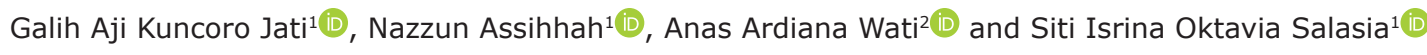

1. Department of Clinical Pathology, Faculty of Veterinary Medicine, Universitas Gadjah Mada, Yogyakarta, Indonesia;

2. Department of Pharmaceutical Chemistry, Faculty of Pharmacy, Universitas Gadjah Mada, Yogyakarta, Indonesia.

Corresponding author: Siti Isrina Oktavia Salasia, e-mail: isrinasalasia@ugm.ac.id

Co-authors: GAKJ: galihaji99@mail.ugm.ac.id, NA: nazzunassihhah@mail.ugm.ac.id,

AAW: anas.ardiana.w@mail.ugm.ac.id

Received: 24-10-2021, Accepted: 05-01-2022, Published online: 11-02-2022

doi: www.doi.org/10.14202/vetworld.2022.288-298 How to cite this article: Jati GAK, Assihhah N, Wati AA, Salasia SIO (2022) Immunosuppression by piperine as a regulator of the NLRP3 inflammasome through MAPK/NF- KB in monosodium urate-induced rat gouty arthritis, Veterinary World, 15(2): 288-298.

\begin{abstract}
Background and Aim: Gouty arthritis is a metabolic disorder involving monosodium urate (MSU) crystal deposition as a key initiator of acute inflammation. Dysregulation of thenucleotide-binding oligomerization domain, leucine-rich repeat, and pyrin domain-containing protein 3 (NLRP3) inflammasome is associated with the pathogenesis of gout through the maturation of interleukin-1 $\beta$. Piperine (PIP) is a phytochemical with an anti-inflammatory activity that has the potential as an alternative treatment for gout. In this study, we examined the effect of PIP in immunosuppression of gout inflammation through the regulation of the NLRP3 inflammasome.
\end{abstract}

Materials and Methods: An in silico study was done by pharmacodynamic modeling of PIP in suppressing MSUinduced inflammation through disruption of the NLRP3 inflammasome. In vivo tests, including inflammatory assessment, histopathology, cytology, estimation of lipid peroxidation index, and detection of systemic inflammatory reactants, were performed on two groups using preventive and curative protocols.

Results: In silico studies of molecular docking demonstrated the activity of PIP as a competitive inhibitor of the mitogenactivated protein kinases/nuclear factor-kappa B axis, upstream of the NLRP3 inflammasome. Analysis of gout models with curative and preventive protocols revealed the immunosuppression activity of PIP by reducing inflammatory symptoms, inhibiting tophus formation resulting from NETosis, reducing cartilage erosion, inhibiting leukocyte exudation, suppressing lipid peroxidation index, and inhibiting the production of C-reactive protein.

Conclusion: The results demonstrate the activity of PIP as an immunosuppressant in gout flare. These findings indicate the potential of PIP as a candidate for prophylactic and therapeutic agent for the treatment of gouty arthritis.

Keywords: gout, immunosuppression, monosodium urate, NLRP3 inflammasome, piperine.

\section{Introduction}

Gouty arthritis is characterized by hyperuricemia and the deposition of monosodium urate (MSU) crystals in articular and periarticular tissues in the extremities, which can trigger acute inflammation [1,2]. Acute gouty inflammation is characterized by pain, swelling from edema, and erythema that attacks the joints [3]. Based on the diagnosis and symptoms, the prevalence of gout is approximately $11.9-24.7 \%$ [4]. The incidence of gout increases and may be accompanied by comorbidities [5].

The pathophysiology of gout is a manifestation of the innate immune inflammatory response triggered by MSU microcrystals. MSU accumulation stimulates monocytes, macrophages, and neutrophils

Copyright: Jati, et al. Open Access. This article is distributed under the terms of the Creative Commons Attribution 4.0 International License (http://creativecommons.org/licenses/by/4.0/), which permits unrestricted use, distribution, and reproduction in any medium, provided you give appropriate credit to the original author(s) and the source, provide a link to the Creative Commons license, and indicate if changes were made. The Creative Commons Public Domain Dedication waiver (http://creativecommons.org/ publicdomain/zero/1.0/) applies to the data made available in this article, unless otherwise stated. to secrete cytokines and chemokines, such as interleukin (IL)-1 $\beta$, tumor necrosis factor- $\alpha$ (TNF- $\alpha$ ), IL-8, IL-6, and monocyte chemotactic factor, which further induce and amplify the inflammatory cascade [6]. A previous study has shown that the production of inflammatory mediators by monocytes and macrophages as a result of MSU phagocytosis plays an important role in the initiation of gout pathogenesis [7]. During an acute gout attack, an influx of neutrophils into the tissues accompanies the secretion of pro-inflammatory cytokines by monocytes and macrophages. This results in the appearance of inflammatory symptoms at the onset of acute gout [8]. The nucleotide-binding oligomerization domain, leucine-rich repeat, and pyrin domain-containing protein 3 (NLRP3) inflammasome complex is a vital component of the innate immune regulators. NLRP3 inflammasome dysregulation enhances the pathogenesis of gout [9]. Martinon et al. [10] demonstrated the role of the NLRP3 inflammasome in MSU-induced IL-1 $\beta$ secretion. Activation of the NLRP3 inflammasome in MSU-induced gout involves two signals. The activation of this complex involves primary signaling 
through nuclear factor kappa B (NF-kB) that causes NLRP3 and pro-IL-1 $\beta$ mRNA expression $[11,12]$. Mitogen-activated protein kinases (MAPK) signaling pathways, including JNK/ERK/p38, activate NF- $\mathrm{KB}$ during the regulation of the NLRP3 inflammasome [13]. JNK-1 (MAPK family) also acts on the second signal through Ser198 of NLRP3 and Tyr146 of adapter apoptosis-associated speck-like protein containing a caspase-recruitment domain (ASC) $[14,15]$. Because of its important function, the MAPK/NF- $\kappa B$ axis is a potential target for the NLRP3 inflammasome regulation during gout.

Current medications used by gout patients include colchicine (Col) and nonsteroidal anti-inflammatory drugs (NSAIDs). Gout patients treated with NSAIDs can exhibit kidney intoxication, gastropathy, hyperkalemia, liver function abnormalities, and headaches [1]. Therefore, alternative treatments and anti-inflammatory therapeutic strategies are needed with minimal side effects to optimize the management of gout. Herbal medicines have become alternatives and generated interest in recent decades because of their potential activity and low toxicity [5]. Piperine (PIP) is a phytochemical compound of the Piperaceae family. PIP exhibits immunomodulator, hepatoprotective, anti-carcinogen, anti-inflammatory, anti-oxidant, and anti-microbial effects [16]. Bang et al. [17] reported that PIP has anti-inflammatory and anti-arthritis activity in synoviocytes stimulated with IL-1 $\beta$. PIP inhibits the production of nitric oxide and TNF during its immunosuppressive effect [18]. In addition, PIP is known to inhibit inflammasome-induced LPS-ATP NLRP3 activity in the HK-2 epithelium [19].

This study aimed to evaluate the anti-inflammatory mechanism of PIP and the efficacy of PIP as an anti-gout agent through preventive and curative action in an MSU-induced gout rat model.

\section{Materials and Methods}

\section{Ethical approval}

All experimental procedures were approved by the Animal Care and Use Committee, Faculty of Veterinary Medicine, Universitas Gadjah Mada (No. 00075/EC-FKH/Int./2021).

\section{Study period and location}

This study was carried out from June to September 2021 at the Clinical Pathology Laboratory of Faculty of Veterinary Medicine and Biochemistry Laboratory of Faculty of Medicine, Public Health, and Nursing, Universitas Gadjah Mada.

\section{Molecular docking of PIP}

A computational approach in the form of molecular docking was made with the help of the 2015 Molecular Operating Environment (MOE) software (Chemical Computing Group, Montreal, Canada). The three-dimension (3D) structure of an inhibitor of nuclear factor-kappa B kinase beta subunit (IKK- $\beta$ )
(PDB ID: 2KIK) and c-Jun N-terminal kinases 1 (JNK1) (PDB ID: 3PZE) were downloaded from the Protein Data Bank website (www.rcsb.org/pdb) [20]. Protein selection was based upon the resolution, source organism, and method of X-ray diffraction. The PIP compound was downloaded from PubChem (https://pubchem.ncbi.nlm.nih.gov/) [21]. Protein preparation, which was done before the docking process, included protonated $3 \mathrm{D}$ and energy minimization. Identifying the binding site was done with the site finder menu available in the MOE software. The affinity strength was obtained after the docking process between the ligand and the target protein was completed.

\section{MSU crystal preparation}

A total of $800 \mathrm{mg}$ of uric acid $\left(\mathrm{C}_{5} \mathrm{H}_{4} \mathrm{~N}_{4} \mathrm{O}_{3}\right)$ (Santa Cruz Biotechnology, Dallas, Texas) were dissolved in $155 \mathrm{~mL}$ of boiling water containing $5 \mathrm{~mL}$ of $1 \mathrm{~N}$ $\mathrm{NaOH}$ (Merck, Darmstadt, Germany). After adjusting the $\mathrm{pH}$ to 7.2 , the solution was cooled for $24 \mathrm{~h}$ at $4^{\circ} \mathrm{C}$. The MSU crystal precipitate was then evaporated and sterilized by heating at $120^{\circ} \mathrm{C}$ for $3 \mathrm{~h}$. MSU was dissolved in $0.9 \% \mathrm{NaCl}$ solution (Merck) at a concentration of $40 \mathrm{mg} / \mathrm{mL}$ before use. The MSU solution was then cooled for 3 days at $4{ }^{\circ} \mathrm{C}$ before injection [22].

\section{Experimental protocol}

For the in vivo experiment, two protocols were used. Protocol I, which was preventive, was designed to determine the potential of PIP as a prophylactic agent for gout. Protocol II was curative and determined the potential of PIP as a therapeutic agent for gout. For each protocol, four treatment groups $(n=3)$ were used, a sham group with $1.25 \mathrm{~mL}$ of $0.9 \% \mathrm{NaCl}$ solution, negative control with $1.25 \mathrm{~mL}(5 \mathrm{mg}) \mathrm{MSU}$ (40 mg MSU in $1 \mathrm{~mL}$ of $0.9 \% \mathrm{NaCl}$ solution), treatment with MSU+PIP (100 mg/kg BW), and a positive control with MSU+Col $(0.28 \mathrm{mg} / \mathrm{kg}$ BW $)$. PIP (Nootropics, Grandville, USA) was dissolved in dimethyl sulfoxide (Merck,) at a $60 \mathrm{mg} / \mathrm{mL}$ concentration and Col (Pratapa Nirmala, Jakarta, Indonesia) was dissolved in distilled water at a concentration of $0.5 \mathrm{mg} / \mathrm{mL}$. In protocol I, PIP and Col were administered orally every day at 9:00 AM for 3 days. MSU injection was performed $1 \mathrm{~h}$ following PIP and Col administration on day 3. Injection of $\mathrm{NaCl}$ solution in the sham group was done simultaneously with MSU. In protocol II, MSU and $\mathrm{NaCl}$ solution injection were carried out once at the beginning of the experiment at 8:00 AM, followed by oral PIP and Col $1 \mathrm{~h}$ after injection. Administration of PIP and Col in protocol II was done for 3 days. Injection of MSU and saline solution was done at the intra-plantar region of the left leg.

\section{In vivo gout induction}

Twenty-four male Wistar rats $(300 \pm 30 \mathrm{~g})$ were divided into two protocols (curative and preventive protocols). Each protocol consists of four groups, namely sham, MSU, MSU+PIP, and MSU+Col ( $\mathrm{n}=3$ per group). Food was provided according to laboratory standards, and drinking water was available $a d$ 
libitum for 20 days of the experiment. Acclimatization was carried out for 7 days before the experiment. The rats were placed in a room with a light-dark cycle of $12 \mathrm{~h}$. Weighing was done before the treatment based on the experimental protocol. Before injection, the rats were first anesthetized with a mixture of $0.1 \mathrm{~mL}$ of ketamine (Agricareperu, Salamanca, Spain) (2 mg/ $\mathrm{mL}$ ) and $0.1 \mathrm{~mL}$ of distilled water. Injection of $1.25 \mathrm{~mL}$ of $0.9 \% \mathrm{NaCl}$ solution (sham group) and $1.25 \mathrm{~mL}$ of MSU (MSU, MSU+PIP, MSU+Col group) was done in the intra-plantar region of the left leg.

\section{Inflammation assessment}

The assessment of rat paw inflammation was performed by measuring the plantar circumference of the foot in the same position, using the tie line method, and scoring. Rat paw inflammation assessment was carried out $1 \mathrm{~h}$ before the injection of $\mathrm{MSU}$ and $0.9 \% \mathrm{NaCl}$ solution. For protocol I, repeated measurements were made at 2, 4, 8, 12, and $24 \mathrm{~h}$ after injection. For protocol II, repeated measurements were made at 2, 4, 8, 12 , 24,48 , and $72 \mathrm{~h}$ after injection. Measurements with the tie line method were carried out in triplicate. Percent edema was measured as swelling rate $=\{(\mathrm{b} / \mathrm{a})-1\} \times 100 \%$, where "a" was the circumference before MSU injection and " $b$ " was the circumference after MSU injection [6]. Scoring was done macroscopically on a scale of $1-3$, where $0=$ no inflammation, $1=$ mild inflammation $2=$ moderate inflammation, and $3=$ severe inflammation [23]. After the rat paw assessment, the blood was collected and centrifuged at $7000 \mathrm{~g}$ for $10 \mathrm{~min}$ using centrifuge PLC-01 (Gemmy Industrial, Taipei, Taiwan), and the serum was separated. The rats were then anesthetized usingketamine and euthanized by decapitation. Necropsy was done by taking plantar (part of the paw) samples of the right foot, liver, and spleen. Plantar samples were fixed in 10\% formalin (Leica, Melbourne, Australia). In contrast, the liver and spleen were homogenized in phosphate-buffered saline (PBS, $\mathrm{pH} 7.2$ ), then cooled at $4^{\circ} \mathrm{C}$ to prepare the tissue homogenate. Serum, foot samples, and homogenized supernatant were then further assessed for histological, cytological, and biochemical test.

\section{Histopathological observation}

Rat right paws were fixed in $10 \%$ formalin were then prepared forhistological analysis by hematoxylin and eosin (H\&E) staining (Leica, Melbourne, Australia). The fixed tissue was decalcified with $0.5 \mathrm{M}$ ethylenediaminetetraacetic acid, $\mathrm{pH} 7.4$ (SigmaAldrich, Missouri, USA). The tissue was then embedded in paraffin (Leica, Melbourne, Australia) and cut to a thickness of $4 \mu \mathrm{m}$ using a rotary microtome (Thermo Fisher, Massachusetts, USA). The slides were then stained with H\&E. The histopathological changes of the tissues were then analyzed by light microscopy (Olympus, Tokyo, Japan) at 100x and 400x magnification and images were digitally captured at a resolution of $1360 \times 1024$ pixels with an Olympus DP70 digital camera (Olympus, Tokyo, Japan).

\section{Giemsa staining}

The collected rat paw samples were then separated from the skin. An impression smear of plantar tissue was performed to determine the pattern of cell infiltration in the exudate following MSU-induced inflammation. The preparations were fixed with 50\% methanol (Merck) and stained with Giemsa, 1:10/ Giemsa (stock solution, Merck): PBS, pH 7.2. After $30 \mathrm{~min}$, they were rinsed with distilled water and the infiltration pattern was observed by light microscopy (Olympus, Tokyo, Japan) at 100x and 400x magnification and images were digitally captured at a resolution of 1360x1024 pixels with an Olympus DP70 digital camera (Olympus, Tokyo, Japan).

\section{Determination of serum malondialdehyde (MDA)}

For the MDA assay, $4 \mathrm{~mL}$ of thiobarbituric acid reagent (Merck) (20\% acetic acid, 0.8\% thiobarbituric acid) was added to $1 \mathrm{~mL}$ of the serum sample. The mixture was homogenized and incubated at $90^{\circ} \mathrm{C}$ for $80 \mathrm{~min}$ and then cooled at $4^{\circ} \mathrm{C}$ in an ice bath for $10 \mathrm{~min}$. Then, $4 \mathrm{~mL}$ of butanol solution (Merck) were added to the mixture and centrifuged at $3000 \mathrm{x} \mathrm{g}$ for $15 \mathrm{~min}$. The supernatant was collected, and the absorbance was read at $510 \mathrm{~nm}$ using a spectrophotometer (Thermo Fisher).

\section{Liver C-reactive protein (CRP) detection}

The 10\% liver homogenate supernatant was tested for CRP using CRP-latex. A total of $40 \mu \mathrm{L}$ of the sample was dripped onto a card and mixed with $50 \mu \mathrm{L}$ of CRP-latex reagent contains sodium azide $0.95 \mathrm{~g} / \mathrm{L}$ (Glory Diagnostics, Barcelona, Spain). The mixture was placed on a mechanical rotator (Gemmy Industrial, Taipei, Taiwan) at $2.24 \mathrm{~g}$ for $2 \mathrm{~min}$. A qualitative analysis of CRP was performed based on the degree of visible agglutination.

\section{Statistical analysis}

Statistical analysis was performed using GraphPad Prism 9.0 software (GraphPad Software, San Diego, California). The tabulation results of the tie line method, scoring, and MDA data are presented as the mean \pm SD. The normal distribution of the data from samples $(n=3)$ was evaluated using the ShapiroWilk test $(\alpha=0.05)$. Statistical significance was determined by one-way analysis of variance, followed by Tukey's post hoc test for scoring data and Dunnet's test for tie line data. $\mathrm{p}<0.05$ was considered statistically significant. The inflammatory, histopathological, cytological, and CRP assessment data was carried out descriptively.

\section{Results}

PIP suppresses the activity of the NLRP3 inflammasome via the MAPK/NF-KB axis

Modeling of the anti-inflammatory effect of PIP for the inhibition of the pathogenesis of MSU-induced gouty arthritis was done using an in silico study. The results of a molecular docking-based study demonstrated the action of PIP as a competitive inhibitor of 
the JNK-1 and IKK- $\beta$ proteins (Figure-1). PIP interacts hydrophobically with the benzene group of residues Val 40 and Val 158 at the active site of JNK-1 (Figure-1a). Visualization of the in silico study also showed the presence of hydrogen bonds between the PIP carbonyl functional group and the amino acid residue, Asn 263, located at the IKK- $\beta$ active site (Figure-1b). The PIP ligand had a root mean square deviation (RMSD) value of $0.8569 \AA$ against IKK- $\beta$ and $0.6914 \AA$ against JNK-1.

The RMSD value represents a validation measure that shows the comparison value between the conformation of the cocrystal ligand and the computational ligand. Based on the molecular docking results, the PIP bond affinity values for IKK- $\beta$ and JNK- 1 were $-5.7428 \mathrm{kcal} / \mathrm{mol}$ and $-6.0626 \mathrm{kcal} / \mathrm{mol}$, respectively.

\section{PIP reduces inflammation in a plantar of rat model of MSU-induced gout}

Based on the pharmacodynamics of PIP as an anti-inflammatory that disrupts the NLRP3 inflammasome cascade, in vivo experiments were done to demonstrate the preventive (protocol I) and curative (protocol II) action of PIP on anti-arthritis gout resulting from MSU deposition.

The protocol I 24-h post-injection of rats demonstrated the preventive action of PIP activity. PIP protection against gout was associated with the inhibition of MSU crystal-induced immunopathogenesis (Figure-2a, c, e). Consistent with protocol I PIP immunosuppression, experiments performed using in protocol II group also demonstrated an effect of PIP on the repression of MSU-induced gouty arthritis (Figure-2b, $\mathrm{d}, \mathrm{f})$. Morphological analysis of plantar in protocol I rats showed that pre-administration of PIP reduced inflammatory symptoms, including erythema, edema, rapid onset of inflammation within $12 \mathrm{~h}$, and tophus formation in this gout model (Figure-2a). The PIP group also exhibited reduced inflammatory symptoms in protocol II, which indicated a drastic suppressive activity of PIP against MSU-induced inflammation (Figure-2b). Scoring-based quantification of inflammatory symptoms in protocol I $24 \mathrm{~h}$ post-injection suggested the amelioration of gout pathogenesis by the preventive activity of PIP (Figure-2c). Scoring in protocol II rats indicated a curative suppression of gout symptoms at all checkpoints (Figure-2d). Experiments to determine the pattern of inhibition of MSU-induced gout by PIP bioactivity were carried out using the tie line method. The swelling rate value (\%) represents the formation of edema. Based on the plot on the swelling rate graph for protocol I, PIP protection predominately suppressed the progression and onset of gout at all checkpoints as early as $2 \mathrm{~h}$ post-injection (Figure-2e), whereas in protocol II, PIP markedly suppressed inflammation, and edema formation during the pathogenesis of gout at its onset (i.e., $12 \mathrm{~h}$ post-injection, Figure-2f). Taken together, the preventive and curative activity of PIP during gout assessment was between that of the sham and MSU groups. In addition, there was a similarity between MSU+PIP and the MSU $+\mathrm{Col}$ groups in both protocols for MSUinduced gout inhibition.

\section{PIP reduces tophus and synovial destruction in MSU- induced gout}

Histopathological microscopic analysis of plantar tissue samples of protocol I and II rats confirmed the potential of PIP as a prophylactic and therapeutic agent for gout by inhibiting acute inflammation, which was demonstrated by an inflammatory assessment. The oral administration of PIP in both protocols demonstrated the immunosuppressive activity of PIP associated with NLRP3 inflammasome inhibition and suppression of the pathogenesis of MSUinduced gout (Figure-3). Comparison of tophus and polymorphonuclear neutrophil (PMN) infiltration in protocol I groups by histopathology $24 \mathrm{~h}$ post-injection revealed the preventive effects of PIP, which was able to reduce tophus formation and an influx in plantar subcutaneous tissue in the rats. Pre-administration of PIP also minimized MSU deposits in the tophus center (Figure-3a). The efficacy of PIP for the reduction of tophus gout was also observed in protocol II (Figure-3b). PIP preparations at 100× magnification showed a small-sized tophus and minimal MSU deposition compared with the MSU group. PMN invasion was also dramatically suppressed by PIP. Investigation of the metatarsophalangeal joints at $400 \times$ magnification indicated the preventive and curative effects of PIP on cartilage erosion resulting from MSU-induced acute inflammation (Figures-3c and d). Evaluation of

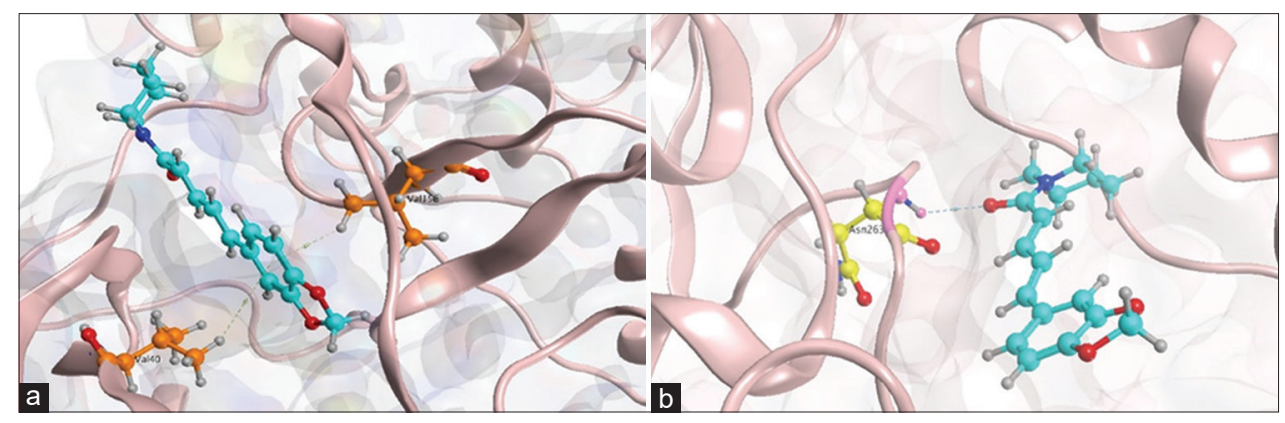

Figure-1: Model of competitive inhibition of piperine (PIP) on the components of the Mitogen-activated protein kinases/ NF-B axis. (a) Visualization of the 3D PIP ligand interaction with the JNK-1 active site. (b) Visualization of the 3D PIP ligand interaction with the active site of inhibitor of nuclear factor-kappa B kinase beta subunit. 


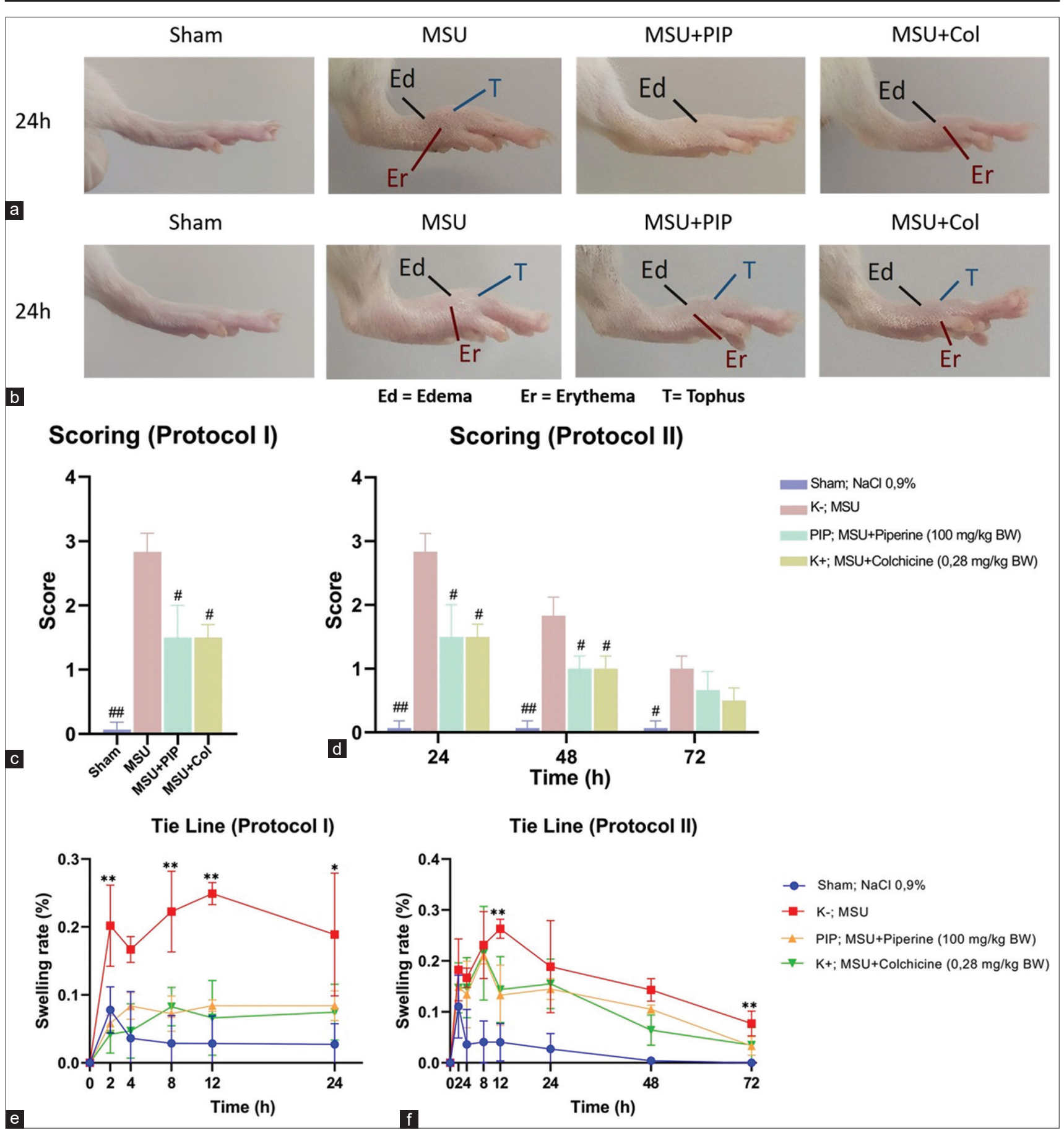

Figure-2: Oral administration of PIP suppresses gouty inflammation induced by injection of monosodium urate (MSU) crystals in rat plantar. ( $a, c, e)$ Anatomical assessment of inflammation in preventive protocol I. (b, d, f) Anatomical assessment of inflammation in curative protocol II (a) comparison of rat paw morphology of protocol I $24 \mathrm{~h}$ post-injection. (b) Comparison of rat paw morphology of protocol II $24 \mathrm{~h}$ post-injection. (c) Protocol I rat plantar inflammation scoring $24 \mathrm{~h}$ post-injection. (d) Protocol II rat plantar inflammation scoring 24, 48, and $72 \mathrm{~h}$ post-injection. (e) The swelling rate of protocol I rat paws was calculated using the tie line method at 2, 4, 8, 12, and $24 \mathrm{~h}$ post-injection. (f) The swelling rate of protocol II rat paws was calculated using the tie line method at 2, 4, 8, 12, 24, 48, and $72 \mathrm{~h}$ post-injection. Values on the graph are represented as mean $\pm S D$ ( $n=3$ rats/group). Significantly different from MSU group, ${ }^{*} p<0.05$, $\# p<0.01$; significantly different from sham, MSU+PIP, MSU+Col groups, $* p<0.05, * * p<0.01$. MSU=Monosodium urate; PIP=Piperine; Col=Colchicine.

protocol I preparations demonstrated the protection of PIP against gout-induced cartilage erosion as observed in the MSU group (Figure-3c). In protocol II group, the proportion of cartilage eroded was higher than that in protocol I. Analysis of protocol II showed the role of PIP in inhibiting cartilage destruction as observed in the MSU group at $72 \mathrm{~h}$ post-injection (Figure-3d).
PIP inhibits leukocyte migration and degeneration in the plantar edema rat gout model

Microscopic analysis of plantar edema in Giemsa-stained rat specimens demonstrated the preventive action of PIP against leukocyte exudation in MSU-induced gout (Figure-4a and c). PIP activity in reducing leukocyte density curatively in gout was also 


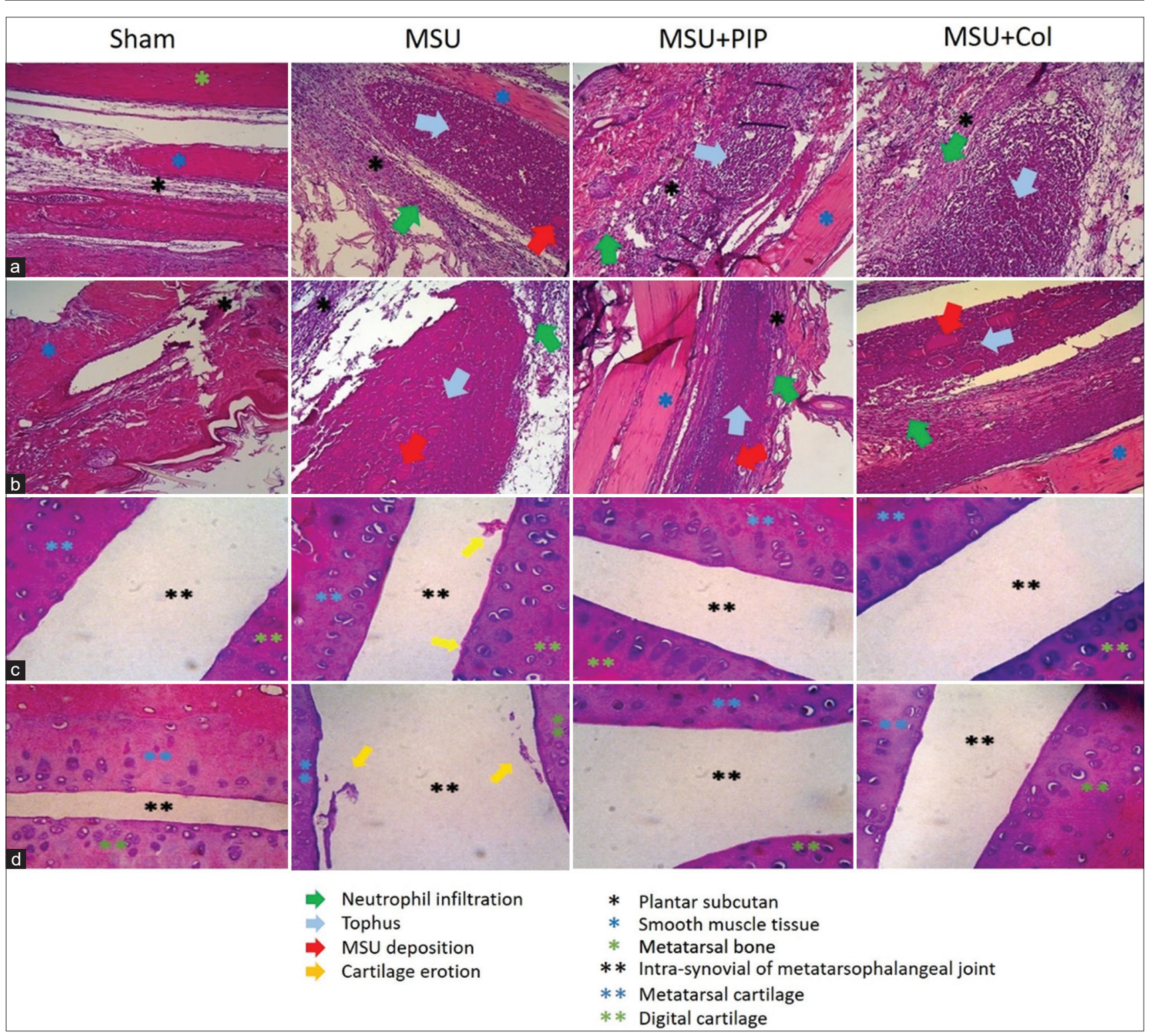

Figure-3: Effects of piperine on the reduction of tophus formation and cartilage erosion in a gout model rat due to intraplantar injection of monosodium urate. ( $a$ and c) Hematoxylin and eosin (H\&E) staining of rat plantar in protocol I. (b and d) H\&E staining of rat plantar in protocol II. (a) Tophus formation and subcutaneous neutrophil infiltration $24 \mathrm{~h}$ post-injection. (b) Tophus formation and subcutaneous neutrophil infiltration $72 \mathrm{~h}$ post-injection. (c) Erosion of the metatarsophalangeal cartilage $24 \mathrm{~h}$ after injection. (d) Erosion of the metatarsophalangeal cartilage $72 \mathrm{~h}$ after injection.

observed in a cytological analysis of edema (Figure- $4 \mathrm{~b}$ and d). Analysis of the plantar edema samples from protocol I rats at $100 \times$ magnification indicated that PIP was protective against monocyte-macrophage exudation. This was observed in the PIP protocol I preparations, in which the monocyte-macrophage density was lower compared with that in the MSU group (Figure-4a). Similar results were also observed for edema in protocol II. PIP reduced monocyte-macrophage exudation through its curative action during MSU-induced inflammation (Figure-4b). The neutrophil influx in gouty edema was observed at 400× magnification. Oral pre-administration of PIP is known to inhibit migration and necroinflammation of degenerative neutrophils (Figure-4c). The curative action of PIP was also evident by the relatively lower neutrophil density of the PIP group compared with the MSU group (Figure-4d). A similar pattern in the reduction of leukocyte density was also found in the orally administered Col group. The samples from both protocols demonstrate PIP pharmacodynamics in the inhibition of the NLRP3 inflammasome cascade that suppresses leukocyte migration and degradation in the exudate during MSU-induced gout inflammation.

\section{PIP inhibits lipid peroxidation in MSU-induced acute gout inflammation}

The quantification of the lipid peroxidation index revealed a decrease in MDA levels in the serum of the PIP-administered gout-afflicted rats (Figure-5). PIP pre-treatment of protocol I rats resulted in protection against elevated serum MDA levels resulting from MSU-induced acute inflammation. PIP inhibition of lipid peroxidation reduced serum MDA levels to a concentration of $15.0 \mu \mathrm{mol} / \mathrm{l}$ (Figure-5a). Evaluation 


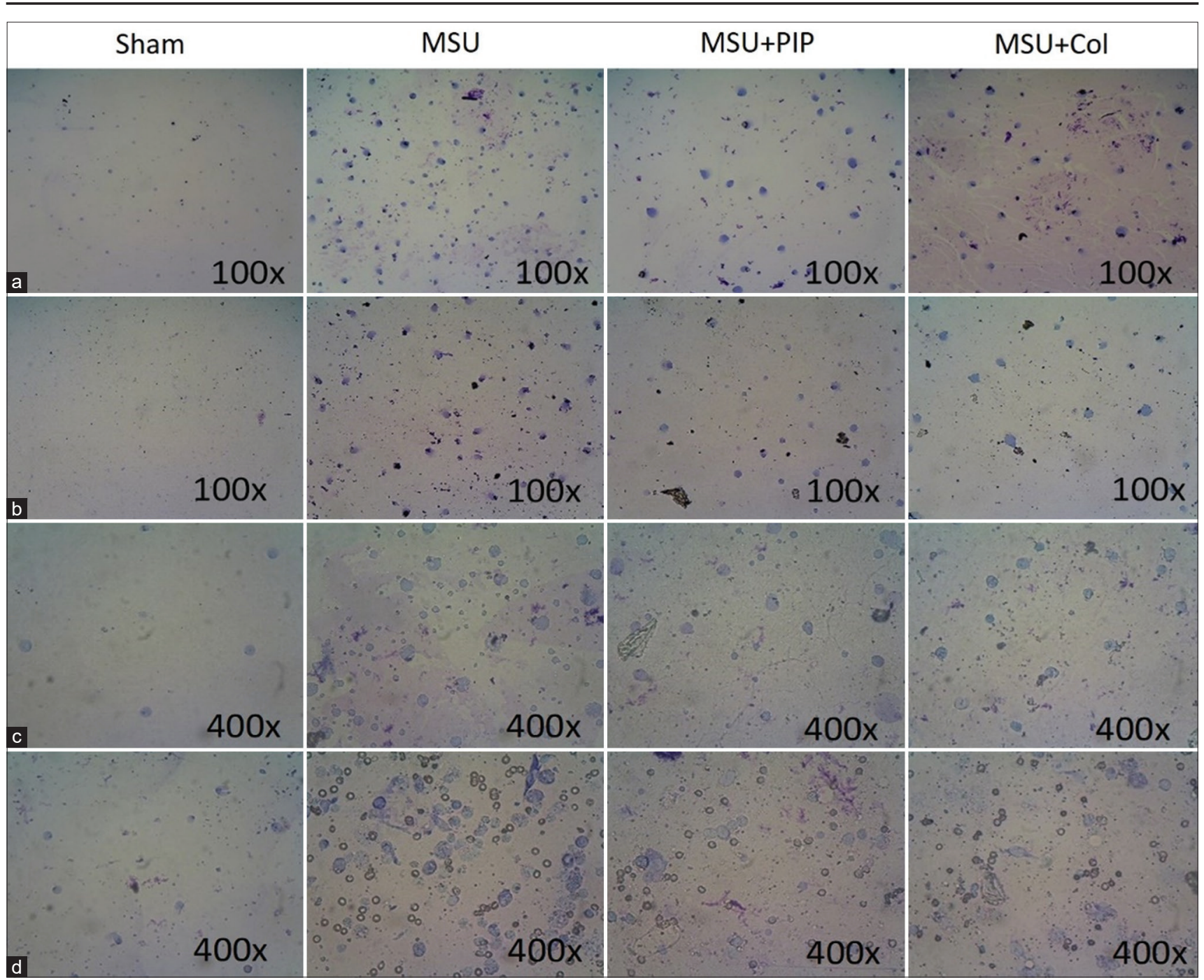

Figure-4: Piperine bioactivity in the inhibition of leukocyte exudation in edema of rat plantar due to monosodium urate injection. ( $a$ and c) Giemsa staining of protocol I rat paw exudate. ( $b$ and d) Giemsa staining of protocol II rat paw exudate. (a) Resident macrophages and monocytes in edema $24 \mathrm{~h}$ post-injection. (b) Resident macrophages and monocytes in edema 72 h after injection. (c) Neutrophil exudation 24 h after injection. (d) Neutrophil exudation 72 h post-injection.

of protocol II MDA levels also indicated a curative action of PIP in the repression of lipid peroxidation from oxidative stress caused by MSU inflammation. PIP activity reduces MDA levels to $19.5 \mu \mathrm{mol}>/ 1$ (Figure-5b). Data from both protocols showed that serum MDA levels of rats administered PIP orally were between MSU and MSU+Col.

\section{PIP reduces hepatic CRP levels resulting from MSU- induced acute inflammation}

Qualitative testing of CRP production by hepatocytes showed a high degree of agglutination in the MSU group compared with the other groups in each protocol. The highest CRP production was observed in the MSU group protocol II samples. This suggests high CRP levels in the liver at $72 \mathrm{~h}$ post-injection because of MSU-induced acute inflammation. Preadministration of PIP in protocol I rats reduced agglutinate density in the rat liver (Figure-6a). Marked PIP inhibition of CRP production was observed in protocol II, in which the agglutinate density of CRP was much lower than that of the MSU group (Figure-6b). Low CRP agglutination was also observed in the MSU+Col group. Analysis of these data indicates the effect of PIP on reducing systemic inflammatory symptoms mediated by CRP reactant in MSU-injected gouty arthritic models. Based on these findings, PIP has the potential to be a prophylactic and therapeutic treatment for the management of the clinical manifestations of gout that are systemic and associated with CRP.

\section{Discussion}

The NLRP3 inflammasome is a multiprotein complex identified as a mediator with an important role in the inflammation of gouty arthritis. It undergoes oligomerization with complex activation following exposure to MSU [24]. The active caspase- 1 conformation of the NLRP3 inflammasome results in the conversion of the pro-IL-1 $\beta$ and pro-IL-18 cytokine precursors to their biologically active forms, IL-1 $\beta$ and IL-18. The proteolytic activity of caspase- 1 also modulates the secretion of pro-inflammatory cytokines during pyroptosis through the Gasdermin D (GSDMD) N-terminal fragment (GSDMD-N) [25]. Activation of the NLRP3 inflammasome in 
MSU-induced gout involves two signals. Primary signaling through TLR 2/4-MyD88-IRAK1/4-TRAF6TAK1-IKK-NF- $\mathrm{KB}$ causes upregulation of NLRP3 and pro-IL-1 $\beta$ mRNA expression [11]. The MAPK signaling pathways, including JNK/ERK/p38, activate NF- $\mathrm{\kappa B}$ for NLRP3 inflammasome regulation when reactive oxygen species production is induced [13]. In addition to acting on the primary signaling pathway, JNK-1 (MAPK family) also acts on the second activation signal through phosphorylation of Ser198 residues in NLRP3 during post-translational modification and Tyr146 of the ASC adapter during speck aggregation $[14,15]$. Because of its central role in regulating the NLRP3 inflammasome, the MAPK/ NF- $\kappa \mathrm{B}$ axis may represent a promising therapeutic target for gout management strategies.

The RMSD value of the PIP ligand $<2$ is considered accurate [26]. The binding affinity values of PIP to IKK- $\beta$ and JNK-1 were $-5.7428 \mathrm{kcal} / \mathrm{mol}$ and $-6.0626 \mathrm{kcal} / \mathrm{mol}$, respectively. The smaller the binding affinity value, the higher the affinity between the

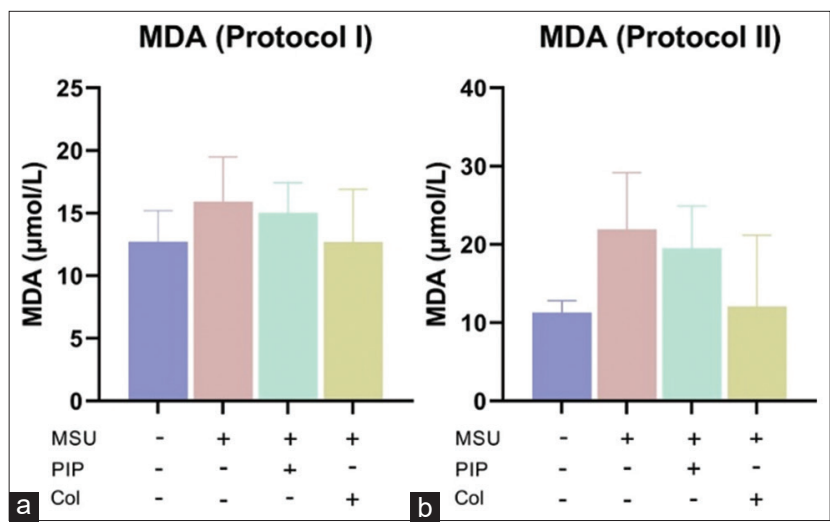

Figure-5: Oral administration of piperine inhibits lipid peroxidation due to monosodium urate-induced gout inflammation, thereby suppressing malondialdehyde (MDA) levels. (a) Serum MDA levels in protocol I. (b) Serum MDA levels in protocol II. Values on the graph are represented as mean $\pm S D$ ( $n=3$ rats/group). ligand and the target protein [26]. Visualization and docking analysis can model the competitive inhibition pattern of PIP on the active site of IKK- $\beta$ and JNK-1. The PIP pharmacodynamic model demonstrates disruption of the MAPK (JNK-1) and NF-KB (IKK- $\beta$ ) signaling pathways, so that they can ameliorate inflammation through the downregulation of the NLRP3 inflammasome.

Gouty arthritis is the most common type of arthropathy and is characterized by inflammation of the joints with MSU as the inducer [1]. Acute inflammation may occur repeatedly during chronic gout and is often referred to as recurrent gout [27]. Metatarsophalangeal articulation is one of the predilections for gout of which patients often complain [2]. Acute inflammation in MSU-induced gout is characterized by a rapid onset, pain, swelling, and erythema [28]. Tophus, which may be palpated as a protrusion in some cases, is an abscess-like cream-textured mass consisting of MSU and degenerative immune cells that can persist and be a predisposition factor for recurrent gout [29]. The rat gout model used in this study was induced by injection of MSU in the plantar of the left foot. MSU is an NLRP3 inflammasome activation factor for the pathogenesis of gout [30]. Col, a first-line drug and an inhibitor of the NLRP3 inflammasome, was used as a reference drug for the in vivo experiments [2,31]. Evaluation of the inflammatory assessment in protocol I indicated that PIP is protective and inhibits the initiation of MSU-induced gout. The potential of PIP as a prophylactic agent may have implications for the inhibition of the pathogenesis of recurrent gout. In addition, the protocol I data may also be the basis for a diet containing PIP as protection against gouty arthritis. Protocol II data also demonstrated the potential of PIP administration as an alternative strategy for the therapeutic management of MSU-induced gout.

NLRP3 inflammasome activation by MSU crystal inducers resulting from phagocytosis by

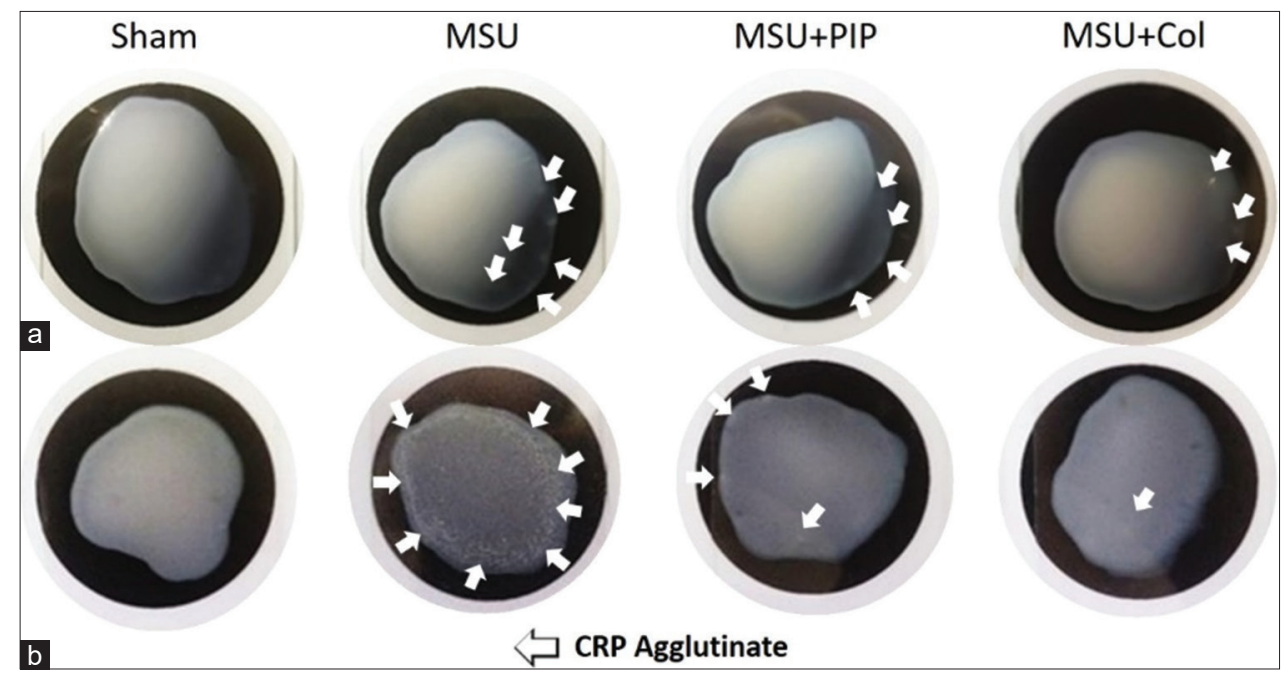

Figure-6: Effect of piperine in reducing C-reactive protein (CRP) in the liver due to monosodium urate-induced inflammation. Detection was carried out based on the agglutination of the $10 \%$ liver homogenate supernatant on CRP-latex. (a) Protocol I (preventive) rat liver CRP agglutinate. (b) Rat liver CRP agglutinate protocol II (curative). 
macrophages and neutrophils may have implications for the inflammatory pathophysiology of gouty arthritis [32]. The production of inflammatory mediators by leukocytes modulated by the NLRP3 inflammasome auto-amplifies necroinflammation, thereby contributing to the progression of gouty arthritis [29]. MSU deposition can induce the PMN invasion of joints during the initiation phase of gout. In addition, the neutrophil influx was identified as a primary marker of pathological gout [6].

Similar characteristics were also observed in our histopathological studies of protocol I (preventive) and protocol II (curative) of gout induced by intra-plantar injection of MSU crystals (Figure-3). The neutrophil influx in gout model lesions resulting from recruitment by resident macrophages phagocytizes MSU and triggers the release of inflammatory mediators. The secretion of cytokines and chemokines, including pleiotropic TNF- $\alpha$ and IL- $1 \beta$, modulates vasodilation, erythema, synovitis, tissue destruction, and cartilage erosion [33]. IL-1 $\beta$ secretion stimulates the release of matrix metalloproteinases enzymes, thereby destroying cartilage and bone and involves osteoblasts [34]. Oval or irregular tophus formation was also observed in hematoxylin- and eosin-stained preparations. Tophus was observed as a crystalline mass of MSU and neutrophil extracellular traps (NETs) similar to granulomas and encapsulated CD68+ macrophages, fibroblasts, and giant cells that contribute to the destruction of surrounding tissues [29].

The ability of PIP to reduce the pathogenicity of acute gouty inflammation, including neutrophil influx, tophus formation, and cartilage erosion, is the basis for a PIP-based gout-arthritis treatment strategy. The lack of MSU deposits on the PIP preparations also indicated the potential of PIP to inhibit recurrent gout, considering that MSU is one of the inducers. In addition, the efficacy of PIP makes it a potential candidate for other diseases associated with immunopathogenesis by PMN invasion and leukocytes contributing to acute inflammation.

The initiation of gout occurs because of the interaction between MSU and resident macrophages, which activate the NLRP3 inflammasome complex, a regulator of the pro-inflammatory cytokine, IL-1 $\beta$ [35]. A previous study has reported that IL-1 $\beta$ plays a central role in the initiation of acute gout [36]. Maturation of IL-1 $\beta$ by the NLRP3 inflammasome induces vasodilation, increased edema formation, leukocyte recruitment, and upregulation of the expression of chemokines and pro-inflammatory cytokines. Edema in MSU-induced gout is associated with leukocyte migration, including monocytes, macrophages, and PMNs, during the process of extravasation by activated endothelial cells [8]. This leukocyte exudation is mediated by endothelial expression of immune cell adhesion molecules, such as E-selectin and vascular cell adhesion molecule 1 [24]. Cytology-based studies of in vivo gout demonstrated the potential of
PIP to inhibit exudation, thereby reducing local edema in MSU-induced gout (Figure-4).

The significant reduction in neutrophil density by PIP also confirmed the ability of PIP to inhibit tophus formation as observed by histopathology in gout samples. These data indicate the preventative and curative activity of PIP for the inhibition of NETosis by neutrophils, which is reported to underlie the formation of aggregated NETs (aggNETs) in tophus [37].

MDA is a marker of lipid peroxidation and it can be used as a parameter in estimating the lipid peroxidation index resulting from oxidative stress during inflammation $[28,38]$. In addition, there is a correlation between the depletion of anti-oxidant activity and the production of MDA by lipid peroxidation. A previous study has reported increased serum MDA levels produced by monocytes, macrophages, and PMNs in an MSU-induced gouty arthritis model [6].

Gout attacks are often accompanied by a systemic inflammatory response, such as fever, where CRP is known to play a role in the manifestation of systemic symptoms of gout arthritis [39]. CRP is a homopentamer protein found in the acute phase of inflammation and is primarily produced by hepatocytes. This substance is also produced by smooth muscle cells, macrophages, endothelium, lymphocytes, and adipocytes [40]. A previous study has demonstrated the key role of CRP during acute inflammation, which involves complement, apoptosis, phagocytosis, and pro-inflammatory cytokines [41]. The pathogenesis of MSU-induced gout involving these components may be initiated and amplified by IL-1 $\beta$, a key regulator of gout maturated by the NLRP3 inflammasome [42]. Therefore, PIP is capable of disrupting the NLRP3 inflammasome and has the potential to inhibit CRP production during the acute phase of gout inflammation.

\section{Conclusion}

The data demonstrated the ability of PIP to suppress inflammation in gouty arthritis by downregulating the NLRP3 inflammasome. The molecular docking studies demonstrated the pharmacodynamic model of PIP as a competitive inhibitor of JNK-1 (MAPK family) and IKK- $1 \beta$ (NF-KB activator), thereby disrupting the oligomerization of the NLRP3 inflammasome, which has implications for inflammation inhibition. The in vivo experiments demonstrated PIP activity in the suppression of MSU-induced gout in a preventive and curative manner. The results indicate the potential of PIP as a prophylactic and therapeutic agent for gouty arthritis.

\section{Authors' Contributions}

SIOS: Conceived, supervised, wrote, and revised the manuscript. GAKJ, NA, and AAW: Research investigation, analyzed the data, wrote, and revised the manuscript. All authors read and approved the final manuscript. 


\section{Acknowledgments}

This study was funded by Ministry of Education and Culture, Republic of Indonesia, through the Student Creativity Program (No. 1949/E2/KM.05.01/2021).

\section{Competing Interests} interests.

The authors declare that they have no competing

\section{Publisher's Note}

Veterinary World remains neutral with regard to jurisdictional claims in published institutional affiliation.

\section{References}

1. Hainer, B.L., Matheson, E. and Wilkes, R.T. (2014) Diagnosis, treatment, and prevention of gout. Am. Fam. Physician, 90(12): 831-836.

2. Li, S., Yang, H. and Chen, X. (2019) Protective effects of sulforaphane on diabetic retino-pathy: Activation of the Nrf2 pathway and inhibition of NLRP3 inflammasome formation. Exp. Anim., 68(2): 221-231.

3. Agudelo, C.A. and Wise, C.M. (2001) Gout: Diagnosis, pathogenesis, and clinical manifestations. Curr. Opin. Rheumatol., 13(3): 234-239.

4. Riset Kesehatan Dasar (Riskesda). (2018) Badan Penelitian dan Pengembangan Kesehatan Kementerian RI tahun 2018. Available from: https://www.litbang.kemkes.go.id/ Laporan-riset-kesehatan-dasar-riskesdas Retrieved on 03-05-2021.

5. Tseuguem, P.P., Nguelefack, T.B., Piégang, B.N. and Mbankou N.S. (2019) Aqueous and methanol extracts of Paullinia pinnata (Sapindaceae) improve monosodium urate-induced gouty arthritis in rat: Analgesic, anti-inflammatory, and anti-oxidant effects. Evid. Based Complement. Alternat. Med., 2019(3): 1-12.

6. Huang, J., Zhu, M., Tao, Y., Wang, S., Chen, J., Sun, W. and $\mathrm{Li}, \mathrm{S}$. (2012) Therapeutic properties of quercetin on monosodium urate crystal-induced inflammation in rat. J. Pharm. Pharmacol., 64(8): 1119-1127.

7. Di Giovine, F.S., Malawista, S.E., Thornton, E. and Duff, G.W. (1991) Urate crystals stimulate production of tumor necrosis factor-alpha from human blood monocytes and synovial cells. J. Clin. Invest., 87(4): 1375-1381.

8. Sabina, E.P., Rasool, M.K., Mathew, L., Ezil, R.P. and Indu, H. (2010) 6-Shogaol inhibits monosodium urate crystal-induced inflammation: An in vivo and in vitro study. Food Chem. Toxicol., 48(1): 229-235.

9. Lin, Y., Luo, T., Weng, A., Huang, X., Yao, Y., Fu, Z., Li, Y., Liu, A., Li, X., Chen, D. and Pan, H. (2020) Gallic acid alleviates gouty arthritis by inhibiting NLRP3 inflammasome activation and pyroptosis through enhancing Nrf2 signaling. Front. Immunol., 11(12): 1-13.

10. Martinon, F., Mayor, A. and Tschopp, J. (2009) The inflammasomes: Guardians of the body. Annu. Rev. Immunol., 27(1): 229-265.

11. Su, H., Li, Y., Hu, D., Xie, L., Ke, H., Zheng, X. and Chen, W. (2018) Procyanidin B2 ameliorates free fatty acids-induced hepatic steatosis through regulating TFEBmediated lysosomal pathway and redox state. Free Radic. Biol. Med., 126: 269-286.

12. Szekanecz, Z., Szamosi, S., Kovács, G.E., Kocsis, E. and Benkő, S. (2019) The NLRP3 inflammasome-interleukin 1 pathway as a therapeutic target in gout. Arch. Biochem. Biophysic., 670(9): 82-93.

13. Zhao, J., Wang, J., Zhou, M., Li, M., Li, M. and Tan, H. (2019) Curcumin attenuates murine lupus via inhibiting NLRP3 inflammasome. Int. Immunopharmacol., 69: $213-216$.
14. Hara, H. (2018) The NLRP6 inflammasome recognizes lipoteichoic acid and regulates gram-positive pathogen infection. Cell, 175(6): 1651-1664.

15. Song, N., Liu, Z.S., Xue, W., Bai, Z.F., Wang, Q.Y., Dai, J., Liu, X., Huang, Y.J., Cai, H., Zhan, X.Y., Han, Q.Y., Wang, H., Chen, Y., Li, H.Y., Li, A.L., Zhang, X.M., Zhou, T. and Li, T. (2017) NLRP3 phosphorylation is an essential priming event for inflammasome activation. Mol. Cell, 68(1): 185-197.

16. Quijia, C. R. and Chorilli, M. (2019) Characteristics, biological properties and analytical methods of piperine: A review. Crit. Rev. Anal. Chem., 50(1): 62-77.

17. Bang, J.S., Oh, H., Choi, H.M., Sur, B.J., Lim, S.J., Kim, J.Y., Yang, H.I., Yoo, M.C., Hahm, D.H. and Kim, K.S. (2009) Anti-inflammatory and antiarthritic effects of piperine in human interleukin 1beta-stimulated fibroblast-like synoviocytes and in rat arthritis models. Arthritis Res. Ther., 11(2): R49.

18. Pradeep, C.R. and Kuttan, G. (2004) Piperine is a potent inhibitor of nuclear factor-kB (NF-kB), c-Fos, CREB, ATF-2 and proinflammatory cytokine gene expression in B16F-10 melanoma cells. Int. Immunopharmacol., 4(14): 1795-1803.

19. Peng, X., Yang, T. and Liu, G. (2018) Piperine ameliorated lupus nephritis by targeting AMPK-mediated activation of NLRP3 inflammasome. Int. Immunopharmacol., 65(7): 448-457.

20. RCSB Protein Data Bank. (2021) Human IkB Kinase Beta. Available from: https://www.rcsb.org/pdb. Retrieved on 03-05-2021.

21. National Center for Biotechnology Information. (2021) PubChem Compound Summary for CID 638024, Piperine. Available from: https://www.pubchem.ncbi.nlm.nih.gov/ compound/Piperine Retrieved 03-05-2021.

22. Miyazawa, K.W., Staurengo-Ferrari, L., Mizokami, S.S., Domiciano, T.P., Vicentini, F.T.M., Camilios-Neto, D., Pavanelli, W.R., Pinge-Filho, P., Amaral, F.A., Teixeira, M.M., Casagrande, R. and Verri, W.A. (2017) Quercetin inhibits gout arthritis in mice: induction of an opioid-dependent regulation of inflammasome. Inflammopharmacology, 25(5): 555-570.

23. Marchetti, C., Swartzwelter, B., Koenders, M.I., Azam, T., Tengesdal, I.W., Powers, N., de Graaf, D.M., Dinarello, C.A. and Joosten, L.A.B. (2018) NLRP3 inflammasome inhibitor OLT1177 suppresses joint inflammation in murine models of acute arthritis. Arthritis Res. Ther., 20(1): 1-11.

24. So, A.K. and Martinon, F. (2017) Inflammation in gout: Mechanisms and therapeutic targets. Nat. Rev. Rheumatol., 13(11): 639-647.

25. He, W.T., Wan, H., Hu, L., Chen, P., Wang, X., Huang, Z., Yang, Z.H., Zhong, C.Q. and Han, J. (2015) Gasdermin D is an executor of pyroptosis and required for interleukin- $1 \mathrm{~b}$ secretion. Cell Res., 25(12): 1285-1298.

26. López-Camacho, E., García-Godoy, M.J., García-Nieto, J., Nebro, A.J. and Aldana-Montes, J.F. (2016) A new multi-objective approach for molecular docking based on RMSD and binding energy. Lect. Notes Comput. Sci., 9702: 65-77.

27. Lim, S.Y., Lu, N., Oza, A., Fisher, M., Rai, S.K., Menendez, M.E. and Choi, H.K. (2016) Trends in gout and rheumatoid arthritis hospitalizations in the United States, 1993-2011. JAMA., 315(21): 2345-2347.

28. Sabina, E.P., Nagar, S. and Rasool, M. (2011) A role of piperine on monosodium urate crystal-induced inflammation an experimental model of gouty arthritis. Inflammation, 34(3): 184-192.

29. Desai, J., Steiger, S. and Anders, H.J. (2017) Molecular pathophysiology of gout. Trends Mol. Med., 23(8): 756-768.

30. Qiao, C.Y., Li, Y., Shang, Y., Jiang, M., Liu, J., Zhan, Z.Y., Ye, H., Lin, Y.C., Jiao, J.Y., Sun, R.H., Zhang, Z.H., Piao, M.H., Wu, Y.L., Nan, J.X. and Lian, L.H. (2020) Management of gout-associated MSU crystals-induced NLRP3 inflammasome activation by procyanidin 
B2: Targeting IL-1 $\beta$ and cathepsin B in macrophages. Inflammopharmacology, 28(6): 1481-1493.

31. Kingsbury, S.R., Conaghan, P.G. and McDermott, M.F. (2011) The role of the NLRP3 inflammasome in gout. $J$. Inflamm. Res., 4(1): 39-49.

32. Goldberg, E.L., Asher, J.L., Molony, R.D., Shaw, A.C., Zeiss, C.J., Wang, C., Morozova-Roche, L.A., Herzog, R.I., Iwasaki, A. and Dixit, V.D. (2017) $\beta$-hydroxybutyrate deactivates neutrophil NLRP3 inflammasome to relieve gout flares. Cell Rep., 18(9): 2077-2087.

33. Schlesinger, N. and Thiele, R.G. (2010) The pathogenesis of bone erosions in gouty arthritis. Ann. Rheum. Dis., 6(11): 1907-1912.

34. Cunninqham, C.C., Corr, E.M. and McCarthy, G.M. (2016) Intra-articular basic calcium phosphate and monosodium urate crystals inhibit anti-osteoclastogenic cytokine signalling. Osteoarthritis Cartilage, 24(12): 2141-2152.

35. Wu M., Zhang, M., Ma, Y., Liu, F., Chen, S., Lu, J. and Chen, H. (2019) Chaetocin attenuates gout in mice through inhibiting HIF-1 $\alpha$ and NLRP3 inflammasome-dependent IL-1 $\beta$ secretion in macrophages. Arch. Biochem. Biophys., 670(1): 94-103.

36. Neogi, T. (2016) Gout. Ann. Intern. Med., 165(1): 1-16.
37. Schauer, C., Janko, C. and Munoz, L.E. (2014) Aggregated neutrophil extracellular traps limit inflammation by degrading cytokines and chemokines. Nat. Med., 20(5): 511-517.

38. Messarah, M. (2010) Influence of thyroid dysfunction on liver lipid peroxidation and anti-oxidant status in experimental rats. Exp. Toxicol. Pathol., 62(3): 301-310.

39. Lee, S.Y., Moon, S.J., Kim, E.K., Seo, H.B., Yang, E.J., Son, H.J., Kim, J.K., Min, J.K., Park, S.H. and Cho, M.L. (2017) Metformin suppresses systemic autoimmunity in Roquinsan/san mice through inhibiting B cell differentiation into plasma cells via regulation of AMPK/mTOR/ STAT3. J. Immunol., 198(7): 2661-2670.

40. Devaraj, S., Singh, U. and Jialal, I. (2009) Human C-reactive protein and the metabolic syndrome. Curr. Opin. Lipidol., 20(3): 182-189.

41. Sproston, N.R. and Ashworth, J.J. (2018) Role of C-Reactive protein at sites of inflammation and infection. Front. Immunol., 9(4): 754-764.

42. Ren, K. and Torres, R. (2009) Role of interleukin-1beta during pain and inflammation. Brain Res. Rev., 60(1): 57-64.

\section{$* * * * * * * *$}

